\title{
Collection Enhancement Mechanism and Test of Side-flow Electrostatic Precipitation
}

\author{
Xiaodong Xiang ${ }^{1,2}$, Siyang Jia ${ }^{3 *}$, Yufeng Chang ${ }^{4}$, Ling Shi ${ }^{4}$ \\ ${ }^{1}$ School of Resource and Environmental Engineering, Wuhan University of Science and Technology, Wuhan 430081, \\ China \\ ${ }^{2}$ Green Manufacturing Engineering Institute, Wuhan University of Science and Technology, Wuhan 430081, China \\ ${ }^{3}$ Hubei Provincial Industrial Safety Engineering Technology Research Center, Wuhan University of Science and \\ Technology, Wuhan 430081, China \\ ${ }^{4}$ Hubei Key Laboratory of Industrial Fume and Dust Pollution Control, Janghan University, Wuhan 430056, China
}

\begin{abstract}
The collection efficiency of many electrostatic precipitators (ESPs) must be improved in order to fulfill the particulate emission standard, which has become increasingly rigorous. According to classical collection theory, decreasing the gas velocity for an electric field is one of the most effective methods of enhancing the collection efficiency. Thus, we proposed a side-flow ESP, which, compared to its conventional counterpart, requires a far lower gas velocity for the electric field when the gas flow rate is constant. First, using aerodynamics, a theoretical expression of the collection efficiency and dust re-entrainment lift force was derived for a turbulent flow. The calculations indicated that a greater increase in efficiency was achieved by decreasing the gas velocity, which potentially reduces dust re-entrainment, than by lengthening the electric field. To confirm the advantages of the side-flow ESP, the collection efficiency and dust re-entrainment were measured in comparison experiments with a conventional ESP, in which talc powder with a medium diameter of $11.6 \mu \mathrm{m}$ was used as the test dust. The dust concentration at the inlet averaged $\sim 1400 \mathrm{mg} \mathrm{m}^{-3}$, and the strength of the electric field ranged approximately from 3 to $4 \mathrm{kV} \mathrm{cm}^{-1}$. The experimental results indicated that the emitted concentration and the dust re-entrainment were $45 \%$ and $42 \%$ lower, respectively, for the side-flow ESP than for the conventional ESP at the same gas flow rate.
\end{abstract}

Keywords: Electrostatic precipitator (ESP); Collection efficiency; Particle penetration; Dust re-entrainment.

\section{INTRODUCTION}

Due to characteristics such as temperature resistance, low-pressure drop, high efficiency, and low operating cost, electrostatic precipitators (ESPs) are widely used in particulate pollutant emission control. Although the collection efficiency of ESPs exceeds $99 \%$ for coarse particles from flue gas, that for collecting fine particles is low (He et al., 2017). The amount of particulates emitted in the air remains high because a large amount of flue gas is produced. According to some studies, total suspended particulate (TSP) emitted from iron and steel industries in China can be reduced by approximately $27-63 \%$ by 2030 under strict environmental standards (Wu et al., 2015). Complying with the increasingly rigorous environmental protection standards is difficult for conventional ESPs. Therefore, improving the collection

\footnotetext{
* Corresponding author.

Tel.: +86 1-862-792-7002

E-mail address: drjsy1914@gmail.com
}

efficiency of dust collection equipment is imperative.

The progress of research in electrostatic collection technology indicated that the main methods used to enhance the dust collection efficiency of ESPs include the optimization of operating conditions, efficient power supply, and improvement of electrode structure (Jaworek et al., 2007). Operating conditions are optimized to increase fine particle collection by changing the physical and chemical properties of flue gas by using methods such as low-low temperature technology (Li et al., 2016), wet electrostatic precipitation (Kim et al., 2014), and gas conditioning (Zhang et al., 2017). To reduce the energy consumption of ESP, inhibit back corona, and improve the efficiency of ESP, the technologies of effective power supply, such as three-phase high-voltage power supply (Jaworek et al., 2015); high-frequency, highvoltage power supply (Soeiro et al., 2013); and high-voltage pulse power supply (Mermigkas et al., 2012) are used. The improvement in electrode structure involves improving the distribution of electric and flow fields by employing the coupled effect of electric power and aerodynamic force, enhancing dust charging, reducing dust re-entrainment, and utilizing electrostatic agglomeration effect. Consequently, 
new ESPs have been developed successively, such as the transverse plate ESP (Yi et al., 2010), wide-spacing ESP (Golkowski and Chojnowski, 1983), electrical agglomeration ESP (Koizumi et al., 2000), moving electrode ESP (Misaka and Mochizuki, 2009), bipolar transverse plate ESP (Xiang et al., 2015), and unipolar pre-charger ESP (Liao et al., 2018). In addition to the methods that enhance the dust collection efficiency, the electrostatically enhanced fabric filtration technology has been widely used for improving the collection efficiency of both new and old ESPs. The types of electrostatic fabric precipitator are as follows: baghouse with positive pre-charger (McCain et al., 1984), stainless steel fiber filters with electric field (Kim et al., 2000), charged porous fibers (Wang et al., 2018), and advanced hybrid particulate collector (Stanley et al., 1999).

In all ESPs developed until now, the gas stream flows straightforwardly from the left inlet duct to the right outlet duct directly, as illustrated in Fig. 1.

When the gas velocity distribution is assumed to be uniform, the collection efficiency of ESPs is given by the Deutsch formula (Deutsch, 1922):

$\eta=1-\exp \left(-\frac{\omega L}{v b}\right)$

$\omega=\frac{q E}{3 \pi \mu d_{p}}$

where $\omega$ is the migration velocity of a charged particle in an electric field; $L$ is the electric field length; $v$ is the average gas velocity in the electric field; $b$ is the distance between electrodes with different polarity; $E$ is the electric field strength; $q$ is the particle charge; $\mu$ is the aerodynamic viscosity; $d_{p}$ is the particle diameter.

Based on Eq. (1), increasing the total length of electric fields is a simple method to enhance the collection efficiency of ESP. However, more space and investment are required. It has been proven that the extent of efficiency improvement cannot be determined using this method because the migration velocity of charged particles does not change. That is, if the fine particles cannot be collected in the front electric field, they also cannot be collected easily in the subsequent electric fields.

According to Eq. (1), a decrease in the gas velocity in the electric field effectively increases collection efficiency. Based on this finding, we proposed a side-flow electrostatic precipitator.

\section{SIDE-FLOW ESP}

The collection efficiency of the ESP can be improved by reducing the gas velocity in the electric field. A decrease in the gas velocity reduces the flow rate of flue gas treatment. The reduced flow rate must not influence the operation requirements of the original pollution control system. However, if the direct flow of flue gas of the conventional ESP is changed to the side flow, decreasing the gas velocity in the electric field is possible, as illustrated in Fig. 2.

In Fig. 2, the flue gas from the left inlet duct enters the inlet chamber first. The gas then turns left at a right angle to pass through electric fields. Finally, the cleaned gas from electric fields enters the outlet chamber and then turns right to the outlet duct.

On comparing the conventional ESP (Fig. 1) with the side-flow ESP (Fig. 2), due to every electric field dimension of both kinds of ESP is the same, no extra space occupied by the side-flow ESP was observed because the total collection electrode area was not changed. The flow rate and residence time of the gas in the electric field were the same. However, the gas velocity in Fig. 2 was decreased to one-third of that in Fig. 1. Moreover, the pressure loss of the side-flow ESP can be considerably lower than that of the conventional ESP because the pressure loss was proportional to the square of the velocity.

To determine the advantages of the side-flow ESP, the effects of the gas velocity on the migration velocity of charged particles and dust re-entrainment must be discussed.

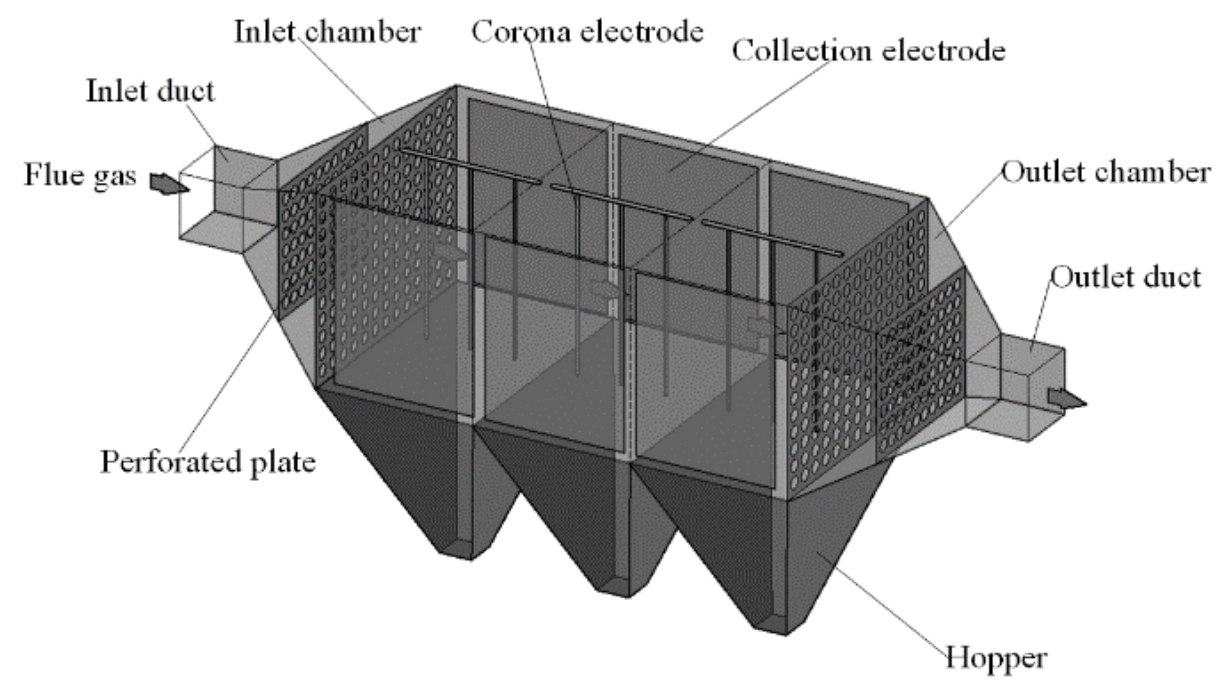

Fig. 1. Gas flow direction in a conventional ESP. 


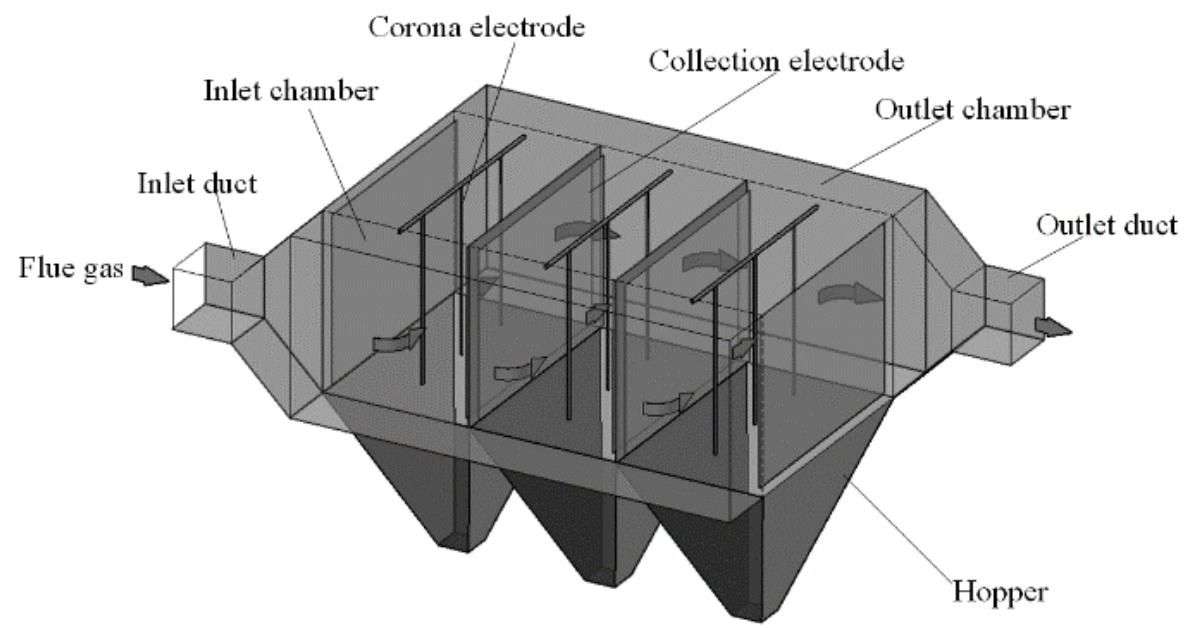

Fig. 2. Gas flow direction in the side-flow ESP.

\section{THEORETICAL ANALYSIS}

\section{Effect of Gas Velocity on Migration Velocity}

When a charged spherical particle $A$, as shown in Fig. 3, enters the electric field between a corona electrode and a collection electrode, an electrical force $F_{E}$ acts on the particle.

$F_{E}=q E$

At the same time, a shear lift force acts on the particle due to gas velocity distribution between electrodes. The shear force $F_{A}$ prevents the charged particle from travelling to the collection electrode and is given by Mei (1992) as:

$F_{A}=C_{L}\left(\frac{\pi d_{p}^{2}}{4}\right) \frac{\rho v^{2}}{2}$

where $\rho$ is the gas density and $C_{L}$ is the lift coefficient, which is determined using the Reynolds number of flow rate around the particle. Reynolds number is given as:

$\operatorname{Re}=\frac{\rho d_{p} v}{\mu}$

Because the average gas velocity in the electric field is approximately $1 \mathrm{~m} \mathrm{~s}^{-1}$, Reynolds number $(\mathrm{Re})$ is usually $<1$. According to Mei's research (Mei, 1992), the lift coefficient $C_{L}$ varies from 2 to 10 .

If the charged particle is moving toward the collection electrode, the drag force acting on it is given by Stokes law:

$f=3 \pi \mu d_{p} \omega_{e}$

where $\omega_{e}$ is the migration velocity of the charged particle when the shear lift force is considered.

Subsequently, the force equilibrium equation can be established as:

$F_{E}-F_{A}-f=0$
By substituting Eqs. (3), (4), and (6) in Eq. (7), the migration velocity of the charged particle is obtained as:

$\omega_{e}=\frac{q E}{3 \pi \mu d_{p}}-\frac{C_{L} \rho}{24 \mu} v^{2}=\omega-k_{1} v^{2}$

where $k_{1}=C_{L} \rho / 24 \mu$.

Therefore, when the shear lift force is considered, the collection efficiency can be written as:

$\eta=1-\exp \left[-\frac{\left(\omega-k_{1} v^{2}\right) L}{v b}\right]$

Eq. (9) proves that the efficiency enhancement effect of reducing the gas velocity $v$ in electric field is much stronger than that of increasing the electric field length $L$.

\section{Effect of Gas Velocity on Dust Re-entrainment}

Dust re-entrainment or particle resuspension resulting from turbulent fluid flow is crucial in the collection processes of ESPs. Dust re-entrainment occurs when aerodynamic lift forces become stronger than adhesive forces. Lift forces acting on particles deposited on the plate electrode include the wall-bounded shear force and energy transferred from the turbulent fluid to round particles.

Leighton and Acrivos (Lazaridis, 2014) derived the mean lift force for a sphere with radius $r_{p}$ resting on a flat surface in wall-bounded shear as:

$F_{B 1}=9.22 \rho v^{2} r_{p}^{4} \approx 0.58 \rho d_{p}^{4} v^{2}$

Because the relationship between energy transfer and lift force is complex, no analytical solution has been obtained so far (Lazaridis, 2014). One of the main energy transfer lift forces is due to the difference in pressure between the top and bottom sides of particles, which is discussed herein.

If gas flow between plate electrodes is considered approximately "long channel," the gas velocity distribution 


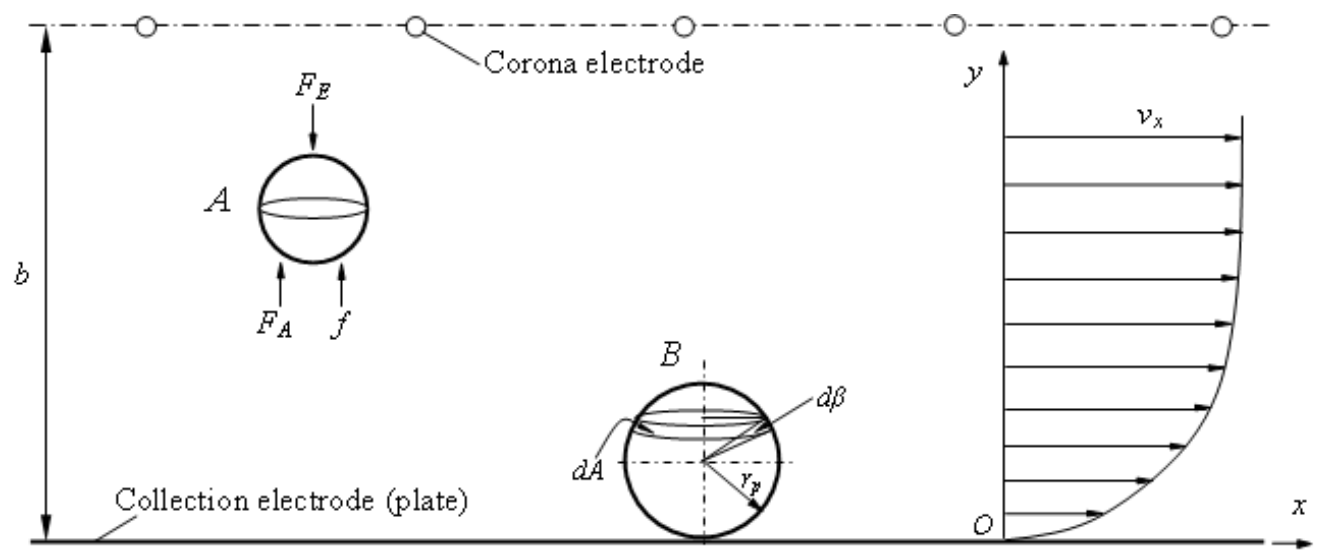

Fig. 3. Gas velocity distribution effects on the migration velocity and dust re-entrainment.

of $v_{x}$ can be expressed as (Crawford, 1976):

$v_{x}=\left(\frac{y}{b}\right)^{1 / n} v_{\max }$

where $v_{\max }$ is the maximum gas velocity in the electric field, and $n$ is approximately $6-9$ in the range of Reynolds number from $5 \times 10^{3}$ to $5 \times 10^{6}$ in turbulent flow. In ESPs, $n$ is approximately 7 .

Based on the Bernoulli equation, the pressure difference in particles deposited on the plate electrode is given as:

$$
p_{s}-p_{\infty}=\frac{1}{2} \rho v_{x}^{2}
$$

where $p_{s}$ is the surface static pressure of the sphere. $p_{\infty}$ is the pressure far away from the particle $B$, as shown in Fig. 3 .

Consider the derivatives $d A$ and $d \beta$ of particle $B$ are in accordance with Eq. (11); then the velocity at the point on the surface of a sphere can be given as:

$v_{x}=\left(\frac{r_{p}+r_{p} \sin \beta}{b}\right)^{1 / 7} v_{\max }$

The area derivative $d A=2 \pi r_{p} \cos \beta \times r_{p} d \beta$. The lift force caused by pressure difference is given as:

$F_{B 2}=\int_{A}\left(p_{s}-p_{\infty}\right) \sin \beta d A=\int_{-\pi / 2}^{\pi / 2} \frac{1}{2} \rho v_{x}{ }^{2}\left(2 \pi r_{p}{ }^{2} \cos \beta \sin \beta\right) d \beta$

By substituting Eqs. (12) and (13) in Eq. (14) the solution is obtained as:

$$
F_{B 2} \approx \frac{4}{21} \pi \rho r_{p}^{2}\left(\frac{r_{p}}{b}\right)^{2 / 7} u_{\max }^{2}
$$

Because the relationship between the average velocity and maximum gas velocity is given as: $v=\frac{1}{b} \int_{0}^{b} v_{x} d y=\frac{1}{b} \int_{0}^{b}\left(\frac{y}{b}\right)^{1 / 7} d y=\frac{7}{8} v_{\max }$

by substituting Eq. (16) in Eq. (15), Eq. (15) becomes:

$F_{B 2}=\frac{4}{21} \pi \rho r_{p}^{2}\left(\frac{r_{p}}{b}\right)^{2 / 7}\left(\frac{8}{7} v\right)^{2} \approx \frac{1}{21} \pi \rho d_{p}^{2}\left(\frac{d_{p}}{b}\right)^{2 / 7} v^{2}$

The total lift force for a sphere on the plate electrode is given as:

$F_{B}=F_{B 1}+F_{B 2}=k_{2} v^{2}$

where $k_{2}=0.58 \rho d_{p}^{4}+\frac{\pi}{21} \rho d_{p}^{2}\left(\frac{d_{p}}{b}\right)^{2 / 7}$.

We conclude that the lift force acting on particles resting on the electrode is directly proportional to the square of the gas velocity. That is, decrease in the gas velocity can considerably reduce the dust re-entrainment.

\section{COMPARATIVE EXPERIMENTS}

The experimental devices of the side-flow ESP with the body size of $400 \times 620 \times 285 \mathrm{~mm}^{3}$ and conventional ESP with the body size of $200 \times 1490 \times 285 \mathrm{~mm}^{3}$ are presented in Figs. 4 and 5, respectively. The electrode structures of both the side-flow and conventional ESP are identical. Stainless steel plate electrodes of $620 \mathrm{~mm}$ in length and $300 \mathrm{~mm}$ in width and stainless steel wire corona electrodes with a diameter of $1 \mathrm{~mm}$ were used. The distance between wires and plates was $100 \mathrm{~mm}$.

The BGG-type negative high-voltage power supply $(60 \mathrm{kV} / 5 \mathrm{~mA})$ was used. The $\mathrm{V}-\mathrm{I}$ characteristics of the tested ESP were measured at room temperature, as illustrated in Fig. 6. In the test, the applied voltage was approximately 25 $45 \mathrm{kV}$. Therefore, the corresponding average electric field strength $E$ was approximately $2.5-4.5 \mathrm{kV} \mathrm{cm}^{-1}$.

Talc powder with a median diameter of $11.6 \mu \mathrm{m}$ was used as the dust sample for measuring the collection efficiency and dust re-entrainment. The particle size distribution of the 


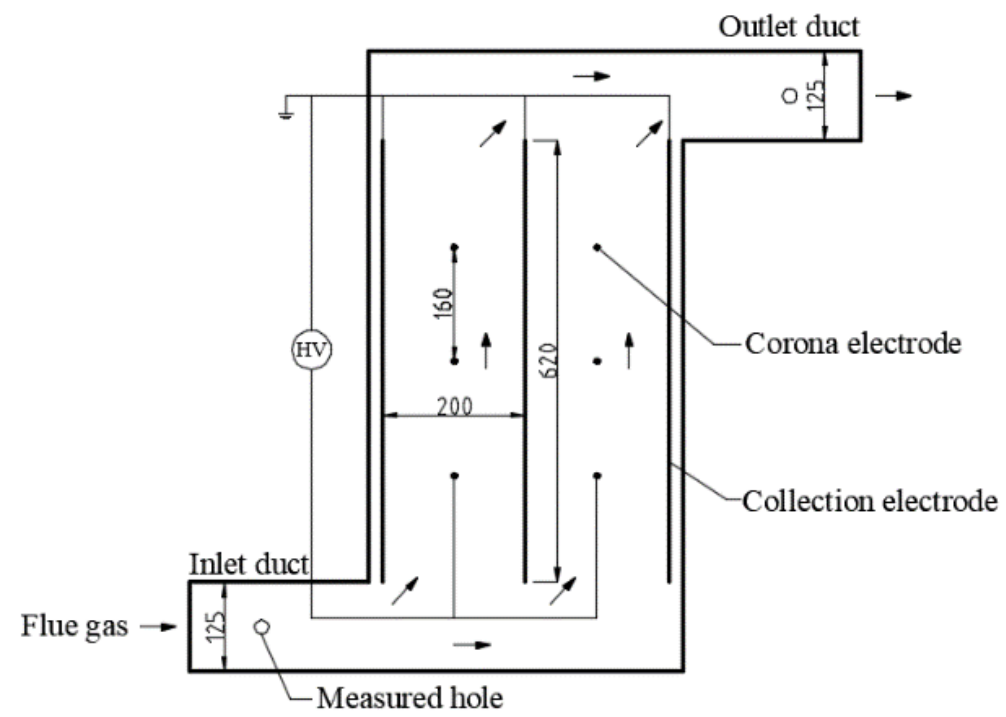

Fig. 4. Test device of the side-flow ESP.

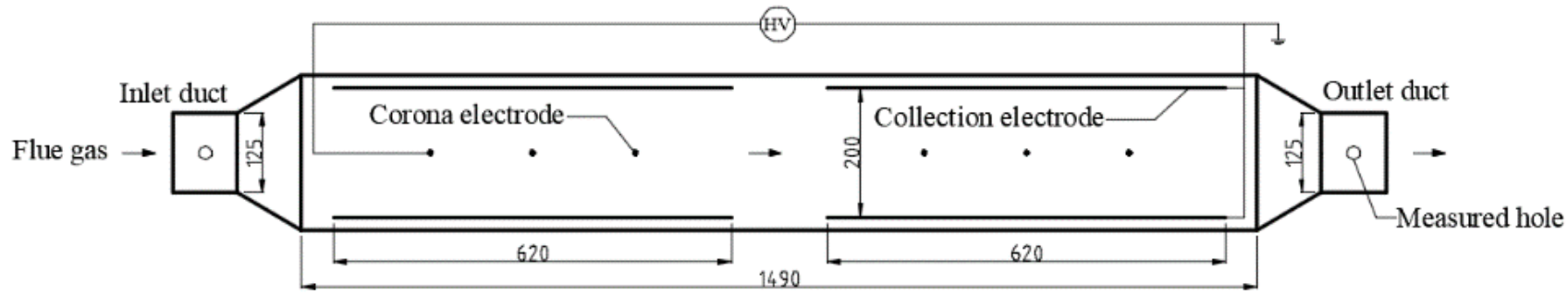

Fig. 5. Test device of the conventional ESP.

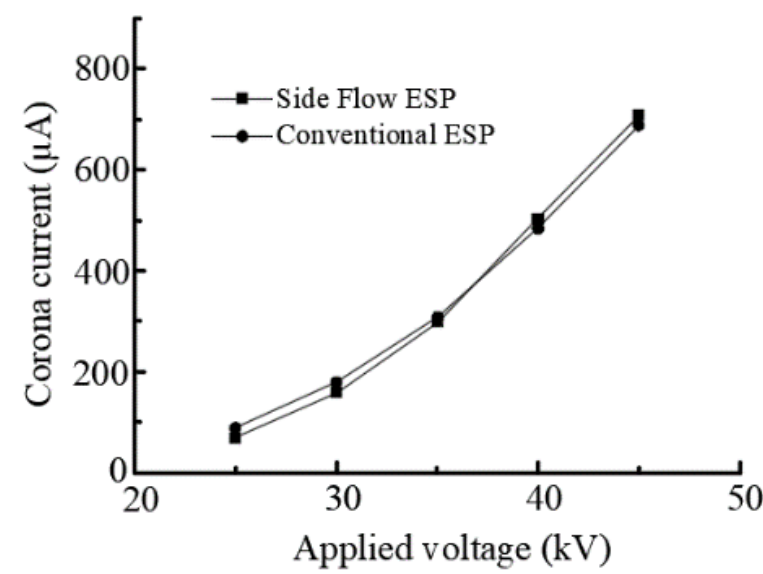

Fig. 6. V-I characteristic of the tested device.

talc powder measured by the MS2000G laser particle size analyzer is presented in Fig. 7.

The dust concentration was measured using a CCD-304 particle concentration detection instrument. The gas velocity was measured using the KA23 anemometer. The experimental gas flow rate was set as $261 \mathrm{~m}^{3} \mathrm{~h}^{-1}$ in both the conventional ESP and side-flow ESP. Corresponding gas velocities in electric fields were 1 and $0.5 \mathrm{~m} \mathrm{~s}^{-1}$ in the conventional and side-flow ESP, respectively. The dust concentration at the inlet was controlled at approximately $1400 \mathrm{mg} \mathrm{m}^{-3}$. The sampling time was 5 minutes.

The comparison tests of the collection efficiency and dust re-entrainment were divided into five groups within the range of electric field strength from 2.5 to $4.5 \mathrm{kV} \mathrm{cm}^{-1}$. In each group, the concentration used was the average value of three times' measuring results.

Because the length of electric field in the side-flow ESP was shorter, conducting the experiments of dust reentrainment during rapping clearing is necessary for determining whether the dust escaped more easily from it. Dust re-entrainment included the following steps:

(1) After every dust collection test, stop producing dust, do not modify the applied voltage and gas flow.

(2) Place a clean filter element in the dust sampling instrument to measure the dust concentration in the outlet.

(3) Use a $700 \mathrm{~g}$ pendulum hammer to strike the bottom frame of the device, as illustrated in Fig. 8.

(4) First, hit the bottom frame 10 times, then remove the filter element from the dust sampling instrument, and finally weigh the filter element to obtain the dust mass.

\section{RESULTS AND DISCUSSION}

\section{Penetration Reduction Ratio}

The comparative experimental results are presented in Table 1, which indicate that the dust collection efficiency of 


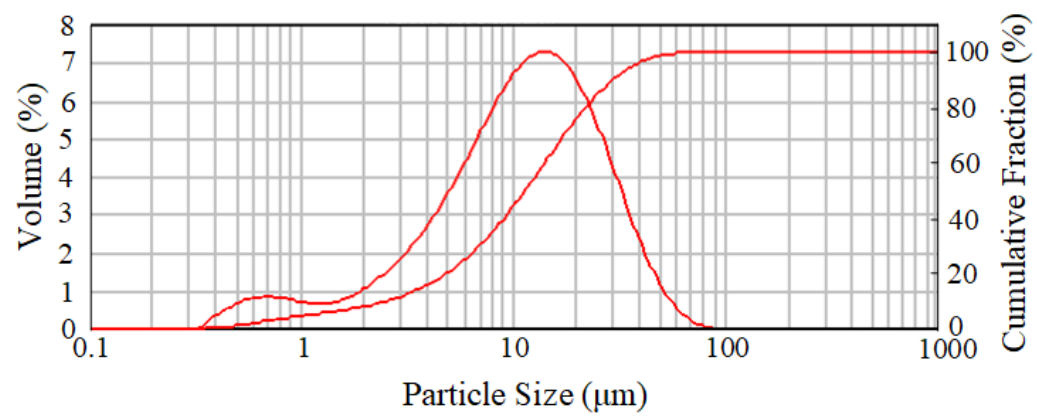

Fig. 7. Particle size distribution of the talc powder.

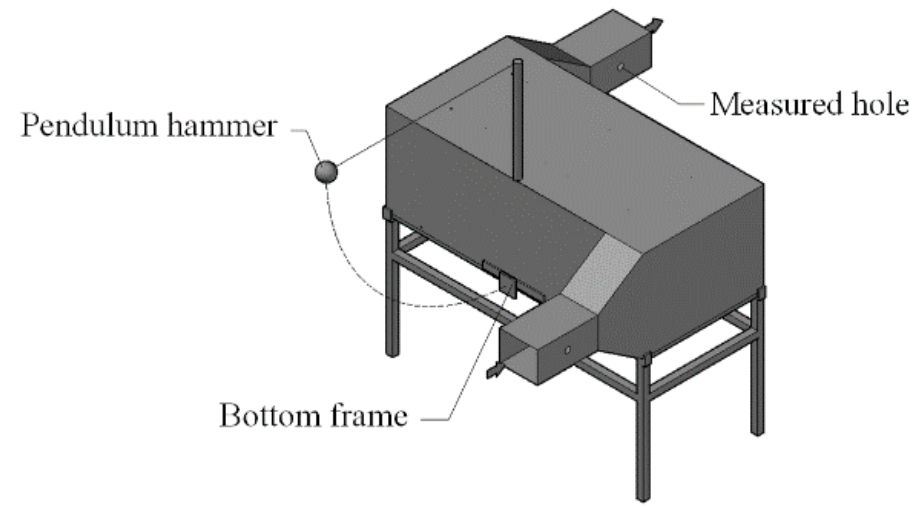

Fig. 8. Electrode rapping, clearing, and measuring techniques in dust re-entrainment experiment.

Table 1. Experimental results of dust collection and re-entrainment of the conventional ESP and side-flow ESP.

\begin{tabular}{|c|c|c|c|c|c|c|c|c|}
\hline $\begin{array}{l}E \\
\mathrm{kV} \mathrm{cm}^{-1}\end{array}$ & ESP & $\begin{array}{l}C_{\text {in }} \\
\mathrm{g} \mathrm{m}^{-3}\end{array}$ & $\begin{array}{l}C_{\text {out }} \\
\mathrm{g} \mathrm{m}^{-3}\end{array}$ & $\begin{array}{l}\eta \\
\%\end{array}$ & $\begin{array}{l}P \\
\%\end{array}$ & $\begin{array}{l}\lambda \\
\%\end{array}$ & $\begin{array}{l}M \\
\mathrm{mg}\end{array}$ & $\begin{array}{l}\delta \\
\%\end{array}$ \\
\hline \multirow[t]{2}{*}{2.5} & Conventional & 1.320 & 0.177 & 86.59 & 13.41 & 49.52 & 1.3 & 38.46 \\
\hline & Side-flow & 1.358 & 0.092 & 93.23 & 6.77 & & 0.8 & \\
\hline \multirow[t]{2}{*}{3.0} & Conventional & 1.345 & 0.114 & 91.52 & 8.48 & 42.81 & 1.1 & 45.45 \\
\hline & Side-flow & 1.360 & 0.066 & 95.15 & 4.85 & & 0.6 & \\
\hline \multirow[t]{2}{*}{3.5} & Conventional & 1.344 & 0.080 & 94.05 & 5.95 & 40.84 & 0.9 & 33.33 \\
\hline & Side-flow & 1.393 & 0.049 & 96.48 & 3.52 & & 0.6 & \\
\hline \multirow[t]{2}{*}{4.0} & Conventional & 1.356 & 0.067 & 95.06 & 4.94 & 45.34 & 1.0 & 50.00 \\
\hline & Side-flow & 1.405 & 0.038 & 97.30 & 2.70 & & 0.5 & \\
\hline \multirow[t]{2}{*}{4.5} & Conventional & 1.393 & 0.044 & 96.84 & 3.16 & 45.25 & 0.9 & 44.44 \\
\hline & Side-flow & 1.388 & 0.024 & 98.27 & 1.73 & & 0.5 & \\
\hline
\end{tabular}

the side-flow ESP was higher than that of the conventional ESP at any electric field strength $E$. To indicate the emission reduction effect of the side-flow ESP clearly, a concept of penetration reduction ratio $\lambda$ was introduced as:

$\lambda=\frac{P_{A}-P_{B}}{P_{A}} \times 100 \%$

where $P_{A}$ and $P_{B}$ are the particle penetrations of the conventional ESP and side-flow ESP, respectively. The particle penetration $P$ is defined as:

$P=\frac{C_{\text {out }}}{C_{\text {in }}}=(1-\eta) \times 100 \%$ where $\mathrm{C}_{\text {in }}$ and $\mathrm{C}_{\text {out }}$ are the inlet and outlet concentrations, respectively, and $\eta$ is the collection efficiency.

The results presented in Table 1 indicate that the penetration reduction ratio in the range of electric field strength from 2.5 to $4.5 \mathrm{kV} \mathrm{cm}{ }^{-1}$ varied from $40.84 \%$ to $49.52 \%$, with an average of approximately $45 \%$, implying that, under same conditions, the dust emission of the sideflow ESP was approximately $45 \%$ lower than that of the conventional ESP.

\section{Dust Re-entrainment Reduction Ratio}

The test results of dust re-entrainment caused by rapping are also listed in Table 1. The results indicated that the dust re-entrainment of the side-flow ESP was lower than that of the conventional ESP. To describe the reduction effect on 
dust re-entrainment of the side-flow ESP, a concept of dust re-entrainment reduction ratio $\delta$ was introduced as follows:

$\delta=\frac{M_{A}-M_{B}}{M_{A}} \times 100 \%$

where $M_{A}$ and $M_{B}$ are the masses of the dust re-entrained for the conventional ESP and side-flow ESP, respectively.

Dust re-entrainment reduction ratios are listed in Table 1. The average dust re-entrainment reduction ratio was $42.34 \%$. The dust re-entrainment of the side-flow ESP was approximately $42 \%$ lower than that of the conventional ESP.

Both the theoretical analysis and comparative experimental results proved that the collection efficiency and dust reentrainment mainly depended on the gas velocity in electric fields. Because the gas velocity of the side-flow ESP was substantially lower than that of the conventional ESP at the same flow rate, the aerodynamic suspension effects on the migration velocity of charged particles and the re-entrainment of dust in collection electrodes were weaker.

\section{CONCLUSIONS}

Compared to a conventional ESP, the side-flow ESP required a considerably lower gas velocity for the electric field at the same flow rate. The theoretical analysis indicated that decreasing the gas velocity, which highly reduced dust re-entrainment, was more effective than lengthening the electric field in improving the collection efficiency. Moreover, the lift force acting on the particles resting on the electrodes was proportional to the square of the gas velocity. Therefore, the gas velocity for the electric field is one of the key factors in electric precipitation.

The results of our comparison experiments indicated that the emitted dust concentration and the dust re-entrainment of the side-flow ESP were approximately $45 \%$ and $42 \%$ less, respectively, than those of the conventional ESP under the same conditions. Therefore, the lower gas velocity resulted in significantly less particulate pollutant emission and dust re-entrainment.

Notably, the gas flow distribution in the electric field near the inlet was not considered in our study. However, distributing the gas flow equally and evenly in every electric field of the side-flow ESP is crucial, although relatively difficult. Thus, the gas flow distribution in this proposed device is a potential subject for future research.

\section{DISCLAIMER}

I declare that I do not have any commercial or associative interest that represents a conflict of interest in connection with the work submitted.

\section{REFERENCES}

Crawford, M. (1976). Air Pollution Control Theory. Mcgraw-Hill, New York, p. 57.

Deutsch, W. (1922). Bewegung und ladung der elektrizitastrager im zylinderkondensator. Ann. Phys. 373: 335-344.

Golkowski, C. and Chojnowski, M. (1983). The role of electrode spacing in the performance of the electrostatic precipitator. J. Electrostat. 14: 339-348.

He, Z., Dass, E.T.M. and Karthik, G. (2017). Design of electrostatic precipitator to remove suspended micro particulate matter from gas turbine inlet airflow: Part I. Experimental study. J. Aerosol Sci. 108: 14-28.

Jaworek, A., Krupa, A. and Czech, T. (2007). Modern electrostatic devices and methods for exhaust gas cleaning: A brief review. J. Electrostat. 65: 133-155.

Jaworek, A., Marchewicz, A., Krupa, A., Sobczyk, A.T., Czech, T., Antes, T. and Rożnowski, W. (2015). Dust particles precipitation in AC/DC electrostatic precipitator. J. Phys. Conf. Ser. 646: 12031.

Kim, H.J., Han, B., Woo, C.G., Kim, Y.J., Ono, R. and Oda, T. (2014). Performance evaluation of dry and wet electrostatic precipitators used in an oxygen-pulverized coal combustion and a $\mathrm{CO}_{2}$ capture and storage pilot plant. J. Aerosol Sci. 77: 116-126.

Kim, S., Sioutas, C. and Chang, M. (2000). Electrostatic enhancement of the collection efficiency of stainless steel fiber filters. Aerosol Sci. Technol. 32: 197-213.

Koizumi, Y., Kawamura, M., Tochikubo, F. and Watanabe, T. (2000). Estimation of the agglomeration coefficient of bipolar-charged aerosol particles. J. Electrostat. 48: 93101.

Lazaridis, I.C.M. (2014). Aerosol Science Technology and Applications. John Wiley and Sons Ltd, pp. 38-42.

Li, Z., Sun, F., Ma, L., Wei, W. and Li, F. (2016). Lowpressure economizer increases fly ash collection efficiency in ESP. Appl. Therm. Eng. 93: 509-517.

Liao, Z., Li, Y., Xiao, X., Wang, C., Cao, S. and Yang, Y. (2018). Electrostatic precipitation of submicron particles with an enhanced unipolar pre-charger. Aerosol Air Qual. Res. 18: 1141-1147.

McCain, J.D., Kistler, W.G., Pontius, D.H. and Smith, W.B. (1984). Electrostatic enhancement of fabric filter performance. Environ. Sci. Technol. 18: 635-641.

Mei, R. (1992). An approximate expression for the shear lift force on a spherical particle at finite reynolds number. Int. J. Multiphase Flow 18: 145-147.

Mermigkas, A.C., Timoshkin, I.V., MacGregor, S.J., Given, M.J., Wilson, M.P. and Wang, T. (2012). Superposition of $\mathrm{DC}$ voltage and submicrosecond impulses for energization of electrostatic precipitators. IEEE Trans. Plasma Sci. 40: 2388-2394.

Misaka, T. and Mochizuki, Y. (2009). Recent Application and Running Cost of Moving Electrode Type Electrostatic Precipitator. Springer Berlin Heidelberg, Heidelberg, Berlin, pp. 518-552.

Soeiro, T.B., Muhlethaler, J., Linner, J., Ranstad, P. and Kolar, J.W. (2013). Automated design of a high-power high-frequency LCC resonant converter for electrostatic precipitators. IEEE Trans. Ind. Electron. 60: 4805-4819.

Wang, C.T., Tu, T.M., Syu, J.Y., Kuo, C.C., Kuo, P.C., Jhong, Y.R. and Lin, W.Y. (2018). Experimental investigation of the filtration characteristics of charged 
porous fibers. Aerosol Air Qual. Res. 18: 1470-1482.

Wu, X., Zhao, L., Zhang, Y., Zheng, C., Gao, X. and Cen, K. (2015). Primary air pollutant emissions and future prediction of iron and steel industry in China. Aerosol Air Qual. Res. 15: 1422-1432.

Xiang, X., Chang, Y. and Nie, Y. (2015). Investigation of the performance of bipolar transverse plate ESP in the sintering flue control. J. Electrostat. 76: 18-23.

Yi, C., Dou, P., Wu, C., Liu, H. and Qu, W. (2010). Experimental study on a laboratory-scale transverse-plate electrostatic precipitator. Fresenius Environ. Bull. 19:
2472-2479.

Zhang, Y., Liu, X., Xu, Y., Sun, W. and Xu, M. (2017). Investigation of reducing ultrafine particulate matter formation by adding modified montmorillonite during coal combustion. Fuel Process. Technol. 158: 264-271.

Received for review, November 17, 2019

Revised, February 12, 2020 Accepted, February 23, 2020 